4

28

\title{
Isolation of Toxoplasma gondii from the brain of a dog in Australia and its
} biological and molecular characterisation

Sarwat Al-Qassab ${ }^{a}$, Michael P. Reichel ${ }^{\mathrm{a},+}$, Chunlei Su ${ }^{\mathrm{b}}$, David Jenkins ${ }^{\mathrm{c}}$, Craig Hall $^{\mathrm{d}}$, Peter A.Windsor ${ }^{\mathrm{e}}$, J. P. Dubey ${ }^{\mathrm{f}}$, John Ellis ${ }^{\mathrm{a}, *}$

(a)

${ }^{a}$ Department of Medical and Molecular Biosciences and Institute for Biotechnology of Infectious Diseases, University of Technology, Sydney, P. O. Box 123, Broadway, New South Wales 2007, Australia

${ }^{b}$ Department of Microbiology, University of Tennessee, Knoxville, TN 37996-0845, USA

${ }^{c}$ School of Animal and Veterinary Sciences, Charles Sturt University, Locked Bag 588, Wagga Wagga, NSW 2678, Australia

${ }^{d}$ Novartis Animal Health Australasia, Kemps Creek, New South Wales 2171 Australia

${ }^{e}$ Faculty of Veterinary Science, University of Sydney, P. O. Box 3, Camden, New South Wales 2570, Australia

${ }^{f}$ United States department of Agriculture, Agricultural Research Service, Animal and Natural Resources Institute, Animal Parasitic Diseases Laboratory, Building 1001, Beltsville, MD 20705-2350, USA

* Corresponding author: Ph: 61295144161

Fax: 612 95148206.Email: john.ellis@uts.edu.au (John Ellis)

+ Present address: School of Veterinary Science, Adelaide University, South Australia 


\section{ABSTRACT}

32 Toxoplasma gondii was isolated from the brain of a young dog for the first time in

33 Australia. The identity of the parasite was confirmed by PCR, Western blotting,

34 electron microscopy and cat bioassay. Genotyping of the isolate (TgDgAu1) was

35 determined by PCR-RFLP markers that showed it to be a Type II strain. Serology

36 demonstrated the presence of IgM antibodies to T. gondii suggesting the bitch was

37 probably infected during pregnancy and the T. gondii was transmitted to the pups

38 congenitally. We believe this represents the first description of a natural case of

39 congenital transmission of T. gondii in the dog.

40

41

42 Key words: Toxoplasma; Dog; Isolation; Genotyping; Australia; Congenital

43 transmission.

44

45

46

47

48 


\section{Introduction} (1) Toxoplasma gondii is a common parasite of most warm blooded animals and birds. The majority of $T$. gondii strains from different human and animal sources have been divided into three clonal but closely related lineages: I, II and III based on restriction fragment length polymorphism (PCR-RFLP), isoenzyme analysis and microsatellite analyses (Howe and Sibley, 1995; Dardé, 1996; Howe et al., 1997; Lehmann et al., 2000; Ajzenberg et al., 2002). Most recently, high resolution genetic markers were developed and revealed an even greater level of polymorphism within this species than previously thought. In addition "atypical strains” were identified, which are different from the clonal type I, II and III lineages (Su et al., 2006; Dubey et al., 2007e). These atypical lineages could be either recombinants from type I, II and III lineages, or unique diverged lineages. Speculations about the global evolution of T. gondii have led to a world-wide effort to study genetic diversity within this species (Lehmann et al., 2006). Little is known about the type of T. gondii found in Australia. Before the discovery of a related parasite, Neospora caninum in 1988 (Dubey et al., 1989), there were many reports of fatal toxoplasmosis in dogs worldwide, including Australia (Dubey and Beattie, 1988). The main aim of this study was to isolate $N$. caninum from the brains of pups born to a bitch known to be infected with this parasite. However T. gondii was unexpectedly isolated. Consequently we report here the isolation and genetic characterization of T. gondii from a young pup in Australia. Serology showed the bitch was probably infected during pregnancy and the T. gondii was transmitted to the pups congenitally. We believe this represents the first description of a natural infection of $T$. gondii occurring via congenital transmission in the dog. 


\section{Material and methods}

\subsection{Host history}

A female cattle dog, aged 18 months, was previously determined to have a titre of 1:200 in the immunofluorescent antibody test (IFAT, VMRD Inc, USA) to $N$. caninum (Hall et al., 2005). In February 2007, the bitch gave birth to six pups and sera from her and all pups were tested in the IFAT and the commercially available latex agglutination test (LAT) (Eiken, Japan) for $N$. caninum and T. gondii antibodies ,respectively. All sera were negative to $T$. gondii in the LAT ( $<1: 8$ serum dilution), but the female cattle dog had an IFAT titre of $>1: 2800$ to $N$. caninum. All pups were weakly positive at $1 / 50$ in the IFAT to $N$. caninum. Serial bleeds were subsequently obtained from the bitch in two-weekly intervals in July and August of 2007 and demonstrated a persistent IFAT titre of $1 / 1000$ for $N$. caninum yet remained negative for T. gondii using LAT.

\subsection{Preparation of brain and cultivation of the parasite}

All animal research was performed with approval from the University of Technology Sydney and Australian National University Animal Care \& Ethics Committees. The six pups were killed at 14 days of age using barbiturate anaesthesia. Whole brains were removed for attempted protozoal isolation by bioassays in cell culture and mice. Brains were homogenized aseptically in saline $(0.95 \% \mathrm{NaCl})$, trypsinized and overlayed on to African Green Monkey kidney (VERO) cells seeded in $75 \mathrm{~cm}^{2}$ vented flasks. Cells were grown in RPMI-1640 medium (Sigma) supplemented with $50 \mathrm{U} / \mathrm{ml}$ penicillin G, $50 \mu \mathrm{g} / \mathrm{ml}$ streptomycin (Sigma) and 2\% heat-inactivated horse serum (Sigma) at $37{ }^{\circ} \mathrm{C}$ in a $5 \% \mathrm{CO}_{2}$ humidified incubator. Each flask was sub-cultured once after three weeks of inoculation and divided into two. For PCR, a small portion of pup brains were stored in the $-80{ }^{\circ} \mathrm{C}$ freezer.

\subsection{Bioassay in mice}

The brain homogenates were also inoculated subcutaneously into female inbred BALB/c mice (Gore Hill Research Laboratories, Sydney) maintained on drinking water supplemented with dexamethasone (Sigma,1 mg/L, Nicoll et al., 1997) beginning at 2 weeks prior to inoculation until the end of experiment. 
In order to investigate virulence of the tachyzoites isolated into culture, untreated mice were inoculated subcutaneously with1, 100 or 1000 culture-derived tachyzoites. Mice were observed for 2 months.

\subsection{Bioassay in cats}

To exclude the possibility of mixed infection with $N$. caninum, mice were infected with 10,000 cultured tachyzoites of TgDgAu1 and subsequently their brains were fed to five T. gondii-free cats as described (Dubey, 1995); T. gondii oocysts are produced by cats and $N$. caninum oocysts are produced in dogs. Faeces of cats were examined for shedding of T. gondii oocysts 3-21 days post-ingesting mouse tissues.

\subsection{DNA extraction and PCR}

DNA was extracted from the brains of pups, mice and in vitro grown tachyzoites. Tissues were snap frozen with liquid nitrogen and ground to a fine powder with a mortar and pestle. Tissues powdered in this way and tachyzoites were lysed in $2 \mathrm{ml}$ of lysis buffer containing 10mM Tris-Cl (pH 7.6), 100mM EDTA, 1\% SDS containing 40 units of proteinase $\mathrm{K}[50 \mathrm{mg} / \mathrm{ml}]$ (Sigma) at $65^{\circ} \mathrm{C}$ for 4 hours. DNA was purified by extraction with phenol-chloroform followed by ethanol precipitation. The DNA obtained was quantified using a Nanodrop spectrophotometer and stored at $-20^{\circ} \mathrm{C}$.

Various primers were used to confirm the parasite identity and to detect $T$. gondii and N. caninum in cultures and dog and mouse tissues. The primer pairs were Tim3/Tim 11 (Payne and Ellis, 1996); T1/T4 for T. gondii (Burg et al. 1989; Chabbert et al. 2004); and the nested PCR described by Barratt et al. (2008) for N. caninum. All PCR reactions were performed on a PTC-100 Programmable Thermal Controller (MJ Research Inc.). DNA from the isolates Me49 of T. gondii (sourced from ATCC) and NC-Liverpool of $N$. caninum were used as controls.

The PCR product produced from cultured tachyzoites isolated from the pup using Tim3 and Tim11 primer sets was purified applying a QIAquick gel extraction kit (QIAGEN), according to the protocol described by the manufacturer and sequencing was performed by the service provider SUPAMAC (Sydney University). Blast searches were conducted using GenBank. 
143

144

145

146

147

148

149

150

151

152

153

154

155

156

157

158

159

160

161

162

163

164

165

166

167

168

169

170

171

172

173

174

175

\subsection{PCR-RFLP genotyping}

Strain typing of $T$. gondii was performed using ten PCR-RLFP genetic markers including SAG1, SAG2, SAG3, $\beta T U B$, GRA6, c22-8, c29-2, L358, PK1 and Apico using nested PCR and following the published protocol (Su et al., 2006; Dubey et al., 2007e).

\subsection{Immunoblot analysis}

Tachyzoites of Me49, NC-Nowra (N. caninum) and TgDgAu1 were purified from cultures by filtration through $3 \mu \mathrm{m}$ filters (Isopore membrane filter, Millipore) and then centrifuged at $3000 \mathrm{rpm}$ for $10 \mathrm{~min}$, after which the pellet was snap-frozen in liquid nitrogen. The tachyzoites were then resuspended in $0.5 \mathrm{ml} \mathrm{PBS}$ and frozen/thawed three times. The mixture was centrifuged at $10,000 \mathrm{~g}$ for $10 \mathrm{~min}$ and the supernatant containing the soluble fraction was removed and stored at $-80{ }^{\circ} \mathrm{C}$.

Western blotting experiments was performed as follows using tachyzoite extracts and either rat anti T. gondii sera or sera from the female dog as primary antibodies.

Tachzyoites were solubilised in NuPage LDS buffer (Invitrogen) containing $5 \% \beta$-mercaptoethanol and electrophoresed on a NuPage $4-12 \%$ Bis-Tris gel (Invitrogen) using 1x XT MES SDS running buffer (Bio-Rad) for $1 \mathrm{hr}$ at $180 \mathrm{~V}$ in a Bio-Rad minigel apparatus. A SeeBlue plus2 pre-stained standard (Invitrogen) was used as a marker. Proteins were transferred to a $0.45 \mu \mathrm{m}$ PVDF membrane (immobilon-P, Millipore) using a Novex transfer system (100 V for $1 \mathrm{hr}$, which was cooled with ice) and blotting buffer (0.192 M Glycine, 0.025 M Tris and 20\% Methanol). The membrane was stained with Ponceau S to visualise the proteins. The membrane was incubated overnight in $5 \%$ skim milk at $4{ }^{\circ} \mathrm{C}$ to block non-specific antibody binding and then washed three times with cold PBS containing 0.02\% Tween 20 (PBST). All subsequent washing of the membrane was also done using PBST.

Serum from a Wister rat infected with the Me49 strain of T. gondii (bled at 8 weeks post infection) was used as primary antibody (1:1000 dilution in 5\% skim milk) and anti-rat IgG (whole molecule)-alkaline phosphatase conjugated antibody (rabbit; Sigma) was used as secondary antibody (1:1000 dilution in 5\% skim milk). Antibody binding was visualised using the Sigma Fast BCIP/NBT alkaline phosphatase substrate (5-bromo-4-chloro-3-indolyl phosphate/nitro blue tetrazolium; 
176

177

178

179

180

181

182

183

184

185

186

187

188

189

190

191

192

193

194

195

196

197

Sigma) dissolved in water. The membrane was then washed with distilled water and allowed to dry.

Serum from the bitch was also used as primary antibody (1:125 dilution in 5\% skim milk) and anti-dog IgG (whole molecule) alkaline phosphatase conjugated antibody (rabbit, Sigma; 1:500 dilution in 5\% skim milk) and anti-dog IgM alkaline phosphatase conjugated antibody (goat, Bethyl Laboratories Inc.; 1:500 dilution) were used as secondary antibodies. Antibody binding was visualised using the Sigma Fast BCIP/NBT alkaline phosphatase substrate.

\subsection{Electron microscopy}

Tachyzoites of TgDgAu1 were washed in three changes of $0.1 \mathrm{M}$ PBS (pH

7.2), and fixed in $3 \%$ glutaraldehyde at $4{ }^{\circ} \mathrm{C}$ then washed three times with $0.1 \mathrm{M}$ PBS.

The tachyzoites were post-fixed in 1\% osmium tetroxide in the same buffer for 2

hours than washed in PBS and enbloc stained with $2 \%$ aqueous uranyl acetate for 20

minutes. Dehydration in ethanol was used, and the tachzyoites infiltrated and

embedded in LR White resin. Blocks were polymerised overnight at $65{ }^{\circ} \mathrm{C}$. Semi-

thin $(1 \mu \mathrm{m})$ sections were stained with $1 \%$ methylene blue. Ultrathin $(70 \mathrm{~nm})$ sections

were cut using a diamond knife (Drukker) and an ultramicrotome (Ultracut S, Leica).

Sections were mounted on 300 mesh copper grids and stained with $2 \%$ aqueous

uranyl acetate for $20 \mathrm{~min}$ and lead citrate (Reynold's) for $4 \mathrm{~min}$. All samples were viewed and imaged in a Philips CM10 TEM. 
198

199

200

201

202

203

204

205

206

207

208

209

210

211

212

213

214

215

216

217

218

219

220

221

222

223

224

225

226

227

228

229

\section{Results}

\subsection{In vitro isolation of the parasite}

PCR of DNA derived from the female dog sera showed that she contained DNA from both T. gondii and $N$. caninum. Homogenates from the brains of the six pups were individually injected into mice or inoculated into tissue culture.

Tachyzoites were observed in all tissue culture flasks after four weeks post inoculation, but flasks inoculated with tissues of five of the six pups were discarded without characterization of the parasites.. After 60 days, tachyzoites started dividing only in the culture derived from pup3 and the present isolate (called TgDgAu1) is derived from this culture.

\subsection{Mouse infection}

All mice (except one) that were injected with brain tissue from the pups and TgDgAu1 tachyzoites remained healthy. One mouse injected with brain tissue from pup4 showed clinical signs manifest as kyphosis from 55 days post-infection and died one week later. This mouse was later shown by PCR to be infected with $N$. caninum but no parasite was recovered into culture.

T. gondii-like tissue cysts of TgDgAu1 were observed in impression smears from brains of mice infected with 10000 tachyzoites after 25 days post infection.

\subsection{PCR analysis}

PCR of DNA derived from sera of the bitch showed it contained DNA from both T. gondii and N. caninum. PCR of DNA from pup brains showed that all brain tissues contained $T$. gondii but only pup3 and pup4 brains were positive to $N$. caninum. PCR of tissue cultures that were inoculated with brain tissue were positive to T. gondii in all flasks, while all were negative by PCR to N. caninum.

Analyses of the DNA sequences of PCR products (derived using Tim3 and Tim11 primers or T1 and T4 primers) from TgDgAu1 showed them to be identical to T. gondii sequences (e.g. GenBank accession no. L49390 or AF179871) thereby confirming the identity of the new isolate as T. gondii.

\subsection{Cat infection}


All cats fed infected mouse tissues shed T. gondii-like oocysts. DNA isolated

231 from them generated a PCR product using T. gondii-specific primers. Bioassay of

232 oocysts in mice was not performed.

233

234

3.5. Genotyping

235

Genotyping results at all ten loci are summarised in Table 1. TgDgAu1

236

displayed the features of a Type II strain at nine loci, while at the SAG1 locus alleles

237 II and III are identical.

\subsection{Western blotting} Immunoblotting of proteins derived from Me49, NC-Nowra and TgDgAu1were probed with antisera from a rat infected with Me49. The antigen

242 profile (Fig. 1a) showed that TgDgAu1 is similar, but not identical to that obtained

243 from Me49, thereby providing additional evidence that the isolate is T. gondii.

244 Western blotting using sera from the bitch obtained on the day of the euthanasia of its

245 pups, and sera from different bleeds taken over six months, showed the bitch had IgG 246 and IgM antibodies to T. gondii (Fig1b, c).

\subsection{Electron microscopy}

249 Electron microscopy of the tachyzoites of TgDgAu1 showed that they had the

250 characteristic morphology of $T$. gondii with few electron-lucent rhoptries. 


\section{Discussion}

The main aim of this study was to isolate $N$. caninum from the brains of pups born to a bitch known to be infected with this parasite. However T. gondii was unexpectedly isolated giving rise to a tachyzoite population (called TgDgAu1) that was stably maintained in culture.

A serological test (a commercial LAT) showed the bitch was seronegative to

262 T. gondii whilst seropositive to $N$. caninum. Similar results were obtained from sera obtained from the bitch up to six months after the birth of its six pups. However Western blotting and PCR of sera from the dog provided evidence that she was coinfected with both T. gondii and N. caninum. Co-infection with these two parasites has also been reported by others (Dubey et al., 2008). The pups were also seronegative to T. gondii by LAT, although PCR on DNA extracted from their brain tissue showed that all six pups contained T. gondii DNA while only pup3 and pup4 were positive to $N$. caninum (representing a mixed infection). Western blotting using anti-IgG and IgM demonstrated that the cattle dog was probably infected with $T$. gondii during pregnancy (Fig 2b, c). Although transplacental transmission of $T$. gondii in naturally infected dogs has not previously been demonstrated, there is evidence from experimentally-infected dogs that congenital infection does occur

\section{4 (Bresciani et al., 1999).}

There is little information on the genotyping of Australian isolates of T. gondii.

276 Only the TG96 strain isolated from a non AIDS patient (see Sibley and Boothroyd,

277 1992) is reported and was genotyped using PCR-RFLP, allozyme analysis and

278 microsatellite (Sibley and Boothroyd, 1992; Howe and Sibley, 1995; Dardé,

279 1996; Ajzenberg et al., 2002). These studies showed that TG96 stain was avirulent 280 and a Type II (= zymodeme 4 and microsatellite group 2) strain. The relatedness of 281 Australian T. gondii to the rest of the world remains unclear.

Genotyping of T. gondii from Asia has been reported in more detail. Studies on dogs in Sri Lanka revealed four genotypes; one of the Type III lineage plus three other atypical genotypes (Dubey et al., 2007d). Two atypical genotypes were detected in dogs from Vietnam (Dubey et al., 2007c). The Type III lineage from Sri Lanka is identical to a genotype isolated from a dog in Brazil (Dubey et al., 2007b). 
identical to a genotype isolated from dog in Colombia (Dubey et al., 2007a), and also

289 to genotypes isolated from chicken and cat in Brazil and China irrespectively (Dubey

290 et al., 2007e, f) suggesting a South American origin of T. gondii currently found in

291 South East Asia (Lehmman et al., 2006; Dubey et al., 2007c).

In conclusion, this study is the first isolation of T. gondii from the

293 brain of a young dog in Australia. Serology showed the bitch was probably infected

294 during pregnancy and T. gondii was transmitted to the pups congenitally. The

295 genotyping of TgDgAu1 confirmed it to be of the Type II linage. Further studies are

296 needed for a better understanding of the diversity of $T$. gondii from this region.

\section{Acknowledgements}

299

We would like to thank P. Williams of Kemps Creek (NSW) for donating the

300 pups; Dr M. Johnson (UTS) for providing the rat sera and experimental assistance

301 with Western blotting; Dr D. Birch from Macquarie University for her assistance with

302 EM. UTS provided finance. This research was conducted by SAQ towards a PhD at 303 UTS. 
305

306

307

308

309

310

311

312

313

314

315

316

317

318

319

320

321

322

323

324

325

326

327

328

329

330

331

332

333

334

335

336

337

\section{References}

Ajzenberg, D., Banuls, A. L., Tibayrenc, M., Dardé, M. L. 2002. Microsatellite analysis of Toxoplasma gondii shows considerable polymorphism structured into two main clonal groups. Int. J. Parasitol. 32, 27-38.

Barratt, J., Al Qassab, S., Reichel, M. P., Ellis, J. T. 2008. The development and evaluation of a nested PCR assay for detection of Neospora caninum and Hammondia heydorni in feral mouse tissues. Mol. Cell. Probes 22, 228-33.

Bresciani, K. D. S., Costa, A. J., Toniollo, G. H., Sabatini, G. A., Moraes, F. R., Paulillo, A. C., Ferraudo, A. S. 1999. Experimental toxoplasmosis in pregnant bitches. Vet. Parasitol. 86, 143-5.

Burg, J. L., Grover, C. M., Pouletty, P., Boothroyd, J. C. 1989. Direct and sensitive detection of a pathogenic protozoan, Toxoplasma gondii, by polymerase chain reaction. J. Clin. Microbiol. 27, 1787-92.

Chabbert, E., Lachaud, L., Crobu, L., Bastien, P. 2004. Comparison of two widely used PCR primer systems for detection of Toxoplasma in amniotic fluid, blood, and tissues. J. Clin. Microbiol. 42, 1719-22.

Dardé, M. L. 1996. Biodiversity in Toxoplasma gondii. Current Topics in Microbiol. Immunol. 219, 27-41.

Dubey, J. P. 1995. Duration of immunity to shedding of Toxoplasma gondii oocysts by cats. J. Parasitol. 81, 410-5.

Dubey, J. P., Beattie, C. P. 1988. Toxoplasmosis in animals and human, CRC Press Inc., Boca Raton, FL USA.

Dubey, J. P., Carpenter, J. L., Topper, M. J., Uggla, A. 1989. Fatal Toxoplasmosis in Dogs. J. Am. An. Hosp. Assoc. 25 659-64. 
Dubey, J. P., Cortés-Vecino, J. A., Vargas-Duarte, J. J., Sundar, N., Velmurugan, G. V., Bandini, L. M., Polo, L. J., Zambrano, L., Mora, L. E., Kwok, O. C., Smith, T., Su, C. 2007a. Prevalence of Toxoplasma gondii in dogs from Colombia, South America and genetic characterization of T. gondii isolates. Vet. Parasitol. 145, 45-50.

Dubey, J. P., Gennari, S. M., Sundar, N., Vianna, M. C., Bandini, L. M., Yai, L. E., Kwok, C. H., Suf, C. 2007b. Diverse and atypical genotypes identified in Toxoplasma gondii from dogs in São Paulo, Brazil. J. Parasitol. 93, 60-4.

Dubey, J. P., Huong, L. T. T., Sundar, N., Su, C. 2007c. Genetic characterization of Toxoplasma gondii isolates in dogs from Vietnam suggests their South American origin. Vet. Parasitol. 146, 347-51.

Dubey, J. P., Rajapakse, R. P., Wijesundera, R. R., Sundar, N., Velmurugan, G. V., Kwok, O. C., Su, C. 2007d. Prevalence of Toxoplasma gondii in dogs from Sri Lanka and genetic characterization of the parasite isolates. Vet. Parasitol. 146, 341-6.

Dubey, J. P., Sundar, N., Gennari, S. M., Minervino, A. H. H., Farias, N. A. D., Ruas, J. L., dos Santos, T. R. B., Cavalcante, G. T., Kwok, O. C. H., Su, C. 2007e. Biologic and genetic comparison of Toxoplasma gondii isolates in free-range chickens from the northern Para state and the southern state Rio Grande do Sul, Brazil revealed highly diverse and distinct parasite populations. Vet. Parasitol. $143,182-8$.

Dubey, J. P., Zhu, X. Q., Sundar, N., Zhang, H., Kwok, O. C. H., Su, C. $2007 f$. Genetic and biologic characterization of Toxoplasma gondii isolates of cats from China. Vet. Parasitol. 145, 352-6.

Dubey, J. P., Stone, D., Kwok, O. C. H., Sharma, R. N. 2008. Toxoplasma gondii and Neospora caninum antibodies in dogs from Grenada, West Indies. J. Parasitol. 94, 750-1. 
Hall, C. A., Reichel, M. P., Ellis, J. T. 2005. Neospora abortions in dairy cattle: diagnosis, mode of transmission and control. Vet. Parasitol. 128, 231-41.

Howe, D. K., Sibley, L. D. 1995. Toxoplasma gondii comprises three clonal lineages correlation of parasite genotype with human-disease. J. Inf. Dis. 172, 1561-6.

Howe, D. K., Honore, S., Derouin, F., Sibley, L. D. 1997. Determination of genotypes of Toxoplasma gondii strains isolated from patients with toxoplasmosis. J. Clin. Microbiol. 35, 1411-4.

Lehmann, T., Blackston, C. R., Parmley, S. F., Remington, J. S., Dubey, J. P. 2000. Strain typing of Toxoplasma gondii: Comparison of antigen-coding and housekeeping genes. J. Parasitology 86, 960-71.

Lehmann, T., Marcet, P. L., Graham, D. H., Dahl, E. R., Dubey, J. P. 2006. Globalization and the population structure of Toxoplasma gondii. Proc. Nat. Acad. Sci. USA 103, 11423-8.

Nicoll, S., Wright, S., Maley, S. W., Burns, S., Buxton, D. 1997. A mouse model of recrudescence of Toxoplasma gondii infection. J. Med. Micro. 46, 263-6.

Payne, S., Ellis, J. T. 1996. Detection of Neospora caninum DNA by the polymerase chain reaction. Int. J. Parasitol. 26, 347-51.

Sibley, L. D., Boothroyd, J. C. 1992. Virulent strains of Toxoplasma gondii comprise a single clonal lineage. Nature 359, 82-5.

Su, C., Zhang, X., Dubey, J. P. 2006. Genotyping of Toxoplasma gondii by multilocus PCR-RFLP markers: a high resolution and simple method for identification of parasites. Int. J. Parasitol. 36, 841-8. 
406 Figure and Table legends

407

408 Fig. 1. Western blotting using rat anti- T. gondii (A) or dog serum (B,C) and

409 tachyzoite extracts. Arrows point to differences between the two isolates (Dog3 is

410 TgDgAu1). B and C were developed with anti-IgG (B) or anti-IgM (C).

411

412

413 Table 1. Summary of genotyping data for the TgDgAu1 isolate of T. gondii.

414

415

416 


\begin{tabular}{ccccccccccccccc}
\hline Genotype & SAG1 & $5^{\prime}+3^{\prime}$ & SAG2 & SAG3 & BTUB & GRA6 & c22-8 & c29-2 & L358 & PK1 & Apico & Isolate IDs \\
\hline Type I & & I & I & I & I & I & I & I & I & I & I & I & RH88 \\
Type II & II or III & II & II & II & II & II & II & II & II & II & II & PTG \\
Type III & II or III & III & III & III & III & III & III & III & III & III & III & CTG \\
Atypical & I & II & II & III & II & II & II & u-1 & I & u-2 & I & TgCgCa1 \\
Atypical & u-1 & I & II & III & III & III & u-1 & I & I & III & I & MAS \\
Atypical & I & III & III & III & III & III & I & I & I & u-1 & I & TgCatBr5 \\
Type II & II or III & II & II & II & II & II & II & II & II & II & II & TgDgAu1 \\
\hline
\end{tabular}

a The SAG2 marker based on 5'- and 3'-end DNA sequence polymorphisms of SAG2 gene (Howe et al., 1997).

b The SAG2 marker developed recently based on 5'-end DNA sequence of SAG2 gene is able to identify additional alleles often seen in atypical T. gondii strains (Su et al., 2006). 


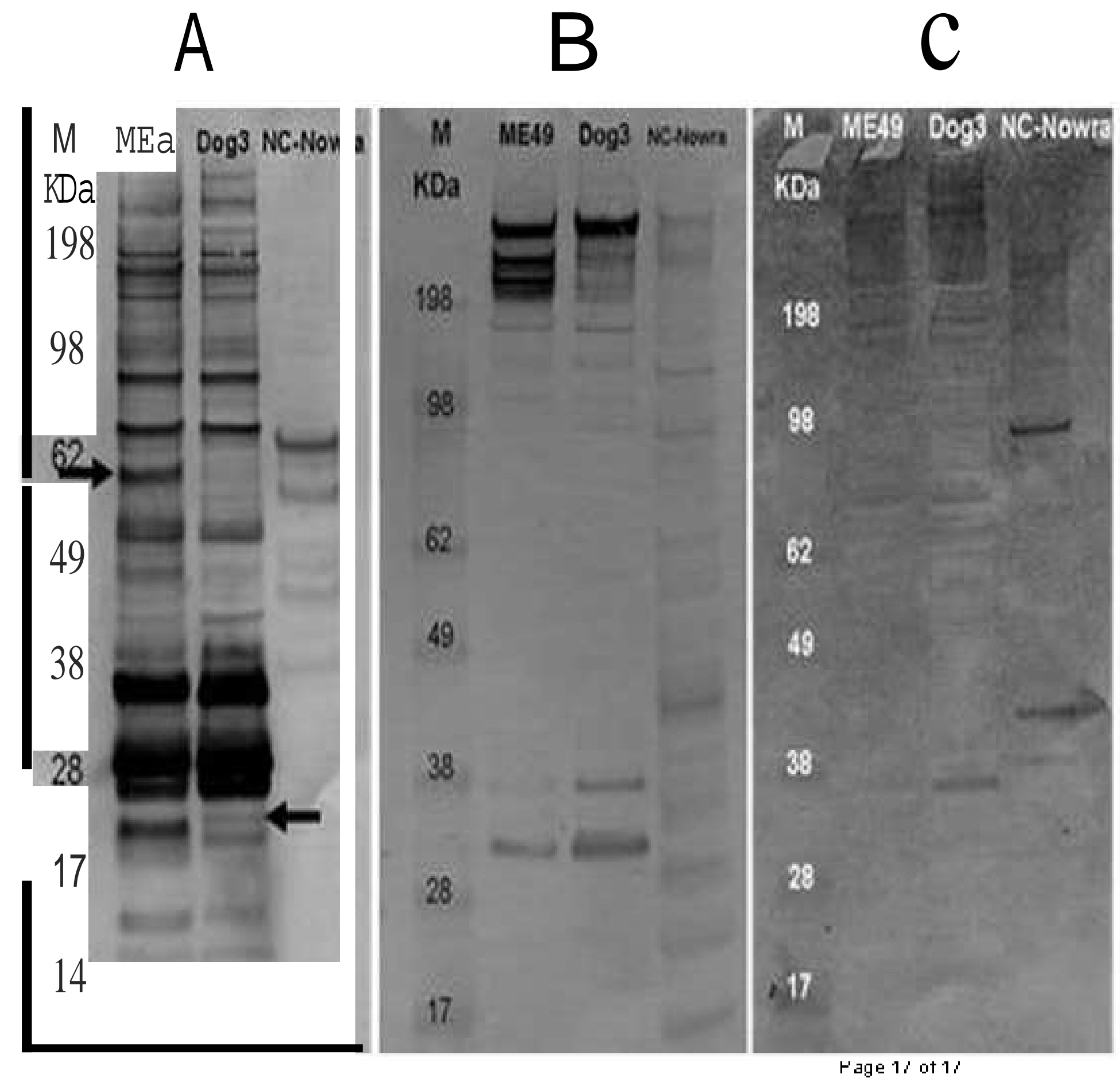

